\title{
Profilaxia do tromboembolismo venoso nas cirurgias do tornozelo e do pé
}

\section{Prophylaxis of Venous Thromboembolism in Ankle and Foot Surgeries}

\author{
1 Pós-Graduação em Ciências Aplicadas à Saúde do Adulto, Faculdade \\ de Medicina, Universidade Federal de Minas Gerais, Belo Horizonte, \\ Minas Gerais, Brasil \\ 2 Serviço de Ortopedia, Rede Mater Dei de Saúde, Belo Horizonte, \\ Minas Gerais, Brasil \\ ${ }^{3}$ Assessoria de Políticas de Saúde e Informações (ASPASI), Hospital \\ Governador Israel Pinheiro (HGIP), Instituto de Previdência dos Servidores \\ do Estado de Minas Gerais (IPSEMG), Belo Horizonte, Minas Gerais, Brasil \\ ${ }^{4}$ Faculdade da Saúde e Ecologia Humana (FASEH), Vespasiano, Minas \\ Gerais, Brasil \\ ${ }^{5}$ Departamento de Medicina Interna, Faculdade de Medicina, Universidade \\ Federal de Minas Gerais, Belo Horizonte, Minas Gerais, Brasil
}

Roberto Zambelli ${ }^{1,2(0)}$ Marcos de Bastos ${ }^{3,4}$ Suely Meireles Rezende ${ }^{5(0)}$

\author{
Endereço para correspondência Roberto Zambelli, PhD, Rua Ouro \\ Preto, 1.016/405, Santo Belo Horizonte, MG, 30.170-041, Brasil \\ (e-mail: zambelliortop@gmail.com).
}

Rev Bras Ortop 2021;56(6):697-704.

\section{Resumo \\ Palavras-chave \\ - trombose \\ - ortopedia \\ - cirurgia \\ - pé \\ - tornozelo \\ - profilaxia pré- exposição}

O tromboembolismo venoso (TEV) é uma das complicações mais temidas pelos ortopedistas, tanto pelo seu desfecho potencialmente letal quanto pelas incertezas relacionadas à sua prevenção. Apesar da vasta literatura existente sobre a prevenção de TEV nas grandes cirurgias ortopédicas, pouco se sabe sobre sua prevenção nas cirurgias do tornozelo e do pé. Uma adequada prescrição da tromboprofilaxia em ortopedia exige criteriosa avaliação dos riscos trombóticos e hemorrágicos com base no tipo de cirurgia a ser realizada, além do conhecimento sobre os anticoagulantes. Esta revisão tem como objetivos abordar a avaliação do risco de desenvolver TEV, as modalidades de tromboprofilaxia, e os fármacos utilizados, tendo como ênfase as cirurgias do pé e do tornozelo.

Venous thromboembolism (VTE) is among the most feared complications by orthopedists due to its potentially lethal outcome and the uncertainties related to its prevention. Despite the vast literature on VTE prevention in major orthopedic surgeries, little is known about it in ankle and foot procedures. In orthopedics, adequate thromboprophylaxis requires a careful assessment of the thrombotic and hemorrhagic risks based on the procedure to be performed, as well as knowledge on anticoagulant agents. The present review has the goal of assessing the risk of developing VTE, the modalities of thromboprophylaxis, and the drugs used, with an emphasis on foot and ankle surgeries. recebido

06 de Março de 2020

aceito

01 de Junho de 2020

Publicado on-line

Setembro 25, 2020
DOI https://doi.org/

10.1055/s-0040-1715512. ISSN 0102-3616. (c) 2020. Sociedade Brasileira de Ortopedia e Traumatologia. All rights reserved.

This is an open access article published by Thieme under the terms of the Creative Commons Attribution-NonDerivative-NonCommercial-License, permitting copying and reproduction so long as the original work is given appropriate credit. Contents may not be used for commercial purposes, or adapted, remixed, transformed or built upon. (https://creativecommons.org/ licenses/by-nc-nd/4.0/)

Thieme Revinter Publicações Ltda., Rua do Matoso 170, Rio de Janeiro, RJ, CEP 20270-135, Brazil 


\section{Introdução}

O tromboembolismo venoso (TEV)é a principal causa de morte evitável no ambiente nosocomial, ${ }^{1}$ e uma das complicações mais conhecidas e temidas das cirurgias ortopédicas. As principais apresentações do TEV são a trombose venosa profunda (TVP) e o tromboembolismo pulmonar (TEP). A primeira pode se complicar com a síndrome pós-trombótica (SPT) e o TEP, e o segundo, com hipertensão pulmonar tromboembólica crônica (HPTC) e morte. ${ }^{2}$ A ocorrência de TEV na população geral é de aproximadamente 1 a 2:1.000 pessoas por ano. ${ }^{3}$

A maioria dos dados referentes à abordagem da prevenção do TEV em ortopedia está relacionada às grandes cirurgias ortopédicas, como as artroplastias de joelho e quadril. ${ }^{4-6}$ As principais diretrizes disponíveis sobre $o$ assunto apresentam recomendação clara e objetiva para que se institua a profilaxia para tais cirurgias, ${ }^{1,4,7}$ incluindo tipo de medicamento e tempo de utilização claramente definidos.

No entanto, para as cirurgias abaixo do nível do joelho, as abordagens e recomendações ainda são obscuras. A diretriz do American College of Chest Physicians (ACCP) sugere que não se use tromboprofilaxia medicamentosa para lesões isoladas abaixo do nível do joelho. ${ }^{4}$ Igualmente, a American Academy of Orthopedic Surgeons declara que não existe uma orientação específica para a profilaxia relacionada às cirurgias do tornozelo e do pé. ${ }^{8}$ Um outro consenso estabelece diretrizes relacionadas aos fatores de risco, mas não define parâmetros objetivos para a indicação de tromboprofilaxia farmacológica, nem o tempo ideal para o seu uso. ${ }^{9}$ A mais recente recomendação da American Society of Hematology nem sequer menciona as lesões de tornozelo e de pé em sua publicação. $^{7}$

O objetivo desta revisão é apresentar dados sobre a incidência de TEV nas cirurgias do tornozelo e do pé, identificar os principais fatores de risco nestas situações, e discutir as ferramentas de predição do risco e as alternativas para tromboprofilaxia.

\section{Incidência}

A ocorrência de TVP nos membros inferiores (TVP-MMII) após uma intervenção ortopédica no nível do tornozelo e do pé é rara, diferente do que se observa nas grandes cirurgias do quadril e do joelho, cuja incidência de TVP-MMII ocorre em $40 \%$ a $60 \%$ dos casos. ${ }^{1}$

Uma meta-análise ${ }^{9}$ com 43.381 pacientes identificou incidência de TEV sintomática de $0,6 \%$ (intervalo de confiança [IC] de $95 \%$ : 0,4\%-0,8\%) em pacientes submetidos a diversas intervenções no pé ou no tornozelo sem tromboprofilaxia, incluindo lesões decorrentes de trauma e intervenções eletivas. Entre os pacientes que receberam algum tipo profilaxia, a incidência foi de 1,0\% (IC95\%: 0,2\%-1,7\%), ${ }^{9}$ possivelmente pelo fato de aqueles pacientes que receberam profilaxia serem pacientes mais graves e/ou com mais fatores de risco para TEV.

Em uma coorte retrospectiva ${ }^{10}$ com 22.486 pacientes submetidos a cirurgia de tornozelo e/ou pé acompanhados entre 2008 e 2011, 173 (0,8\%) tiveram TEV sintomático nos 6 meses subsequentes à intervenção ortopédica. ${ }^{10}$ Quando avaliado o local da cirurgia e a ocorrência de trombose, $65,6 \%$ dos casos de TEV ocorreram em cirurgias do retropé e do tornozelo, $11,1 \%$, no mediopé, e $26,7 \%$, no antepé.

Sabe-se que, entre as doenças do pé e do tornozelo, a ruptura do tendão de Aquiles é a lesão mais frequentemente associada à ocorrência de TEV. Os dados de uma metaanálise ${ }^{9}$ mostram que entre 1.060 pacientes com ruptura do tendão de Aquiles avaliados clinicamente para a investigação de TEV, 74 (7\%) tiveram TEV confirmado. ${ }^{9}$ Entre 1997 e 2015, um estudo ${ }^{11}$ avaliou 28.546 pacientes com ruptura aguda do tendão de Aquiles, tratados cirurgicamente ou não, quanto à incidência de TEV. ${ }^{11}$ Destes, 389 (1,36\%) foram diagnosticados com trombose, dos quais $278(0,97 \%)$ tiveram TVP-MMII, e 138 (0,48\%), TEP. Em um outro estudo de coorte prospectivo, ${ }^{12}$ dos 291 pacientes com ruptura aguda do tendão calcâneo, 14 (4,81\%) foram diagnosticados com TEV nos primeiros 33 dias após o procedimento cirúrgico. ${ }^{12}$

É importante ressaltar que a maioria dos eventos tromboembólicos identificados nos estudos supracitados são assintomáticos, sendo somente detectados por exames de imagem de rastreamento, realizados após o procedimento cirúrgico. Por isso, a maior parte destes eventos são de menor ou nenhuma relevância clínica. Como exemplo, cita-se um estudo de coorte prospectivo, ${ }^{13}$ no qual 114 cirurgias eletivas em pé e/ou tornozelo foram realizadas em 111 pacientes, sendo constatada TVP em 29 (25,4\%), dos quais a maioria (20/29, ou $68,9 \%$ ) foi diagnosticada por ultrassonografia na visita de rastreamento 2 semanas após a cirurgia. ${ }^{13}$ Em outro estudo de coorte prospectivo com 216 pacientes submetidos a abordagens cirúrgicas diversas no pé ou no tornozelo, a TVP foi rastreada por ultrassonografia dos membros inferiores entre 2 e 6 semanas após a cirurgia. A incidência de TEV foi de 5,09\% (11/216), e 3 dos 11 pacientes com TEV tiveram TVP assintomática. ${ }^{14}$ Assim, uma vez que a maioria dos eventos tromboembólicos venosos assintomáticos apresentam pouca repercussão clínica, e que menos de 10\% dos casos das TVPMMII distais sintomáticas apresentam extensão proximal, ${ }^{15} \mathrm{o}$ rastreamento de TEV por ultrassom em pacientes assintomáticos submetidos a cirurgias abaixo do nível do joelho não deve ser realizado na prática clínica. Este exame deve ser indicado para aqueles pacientes com suspeição clínica de TVP. ${ }^{9}$

No entanto, a grande dificuldade em se determinar o risco de TEV nos pacientes com lesões abaixo do nível do joelho decorre dos seguintes fatores: 1) a maioria dos estudos se baseia em série de casos; 2) as informações referentes ao uso de uma eventual tromboprofilaxia nestes estudos é inadequada; 3) devido à baixa incidência, o número de pacientes com TEV nos estudos relatados não foi capaz de oferecer conclusões suficientemente robustas; ${ }^{16}$ e 4 ) a escassez de estudos que tenham avaliado o impacto de fatores de risco associados na ocorrência de TEV nos pacientes com intervenções cirúrgicas abaixo do nível do joelho., 17-19

\section{Fatores de risco}

As cirurgias ortopédicas, principalmente as grandes cirurgias do quadril e do joelho, são procedimentos de alto risco para a ocorrência de TEV. ${ }^{3}$ No entanto, embora o risco trombótico 
seja menor, as intervenções no tornozelo e no pé, que habitualmente requerem algum tempo de restrição de apoio do membro inferior no solo e/ou imobilização, também se associam a maior risco. ${ }^{9,17}$

A imobilização do membro inferior, que impede a contração da musculatura da panturrilha, tem se mostrado um importante fator de risco isolado para TEV nas intervenções abaixo do joelho. ${ }^{20,21}$ Além disso, sabe-se que, quanto maior o tempo de imobilização, maior o risco de TEV. ${ }^{22}$ Adicionalmente, o fato de permanecer sem apoiar o membro inferior no solo também está associado ao aumento do risco de TEV, sendo muitas vezes somado à imobilização como um fator de risco maior. ${ }^{23} \mathrm{O}$ uso de imobilização sem apoio por um período de 2 a 8 semanas está associado a um risco de TEV 9 vezes maior (razão de probabilidades [RP]: 9,0; IC95\%: 1,8-44,3) do que naqueles pacientes que permanecem imobilizados por um período inferior a este. ${ }^{10}$ Apesar de alguns autores considerarem que o fato de poder apoiar o membro inferior no solo possibilita interromper a profilaxia medicamentosa, ${ }^{14}$ por conferir maior mobilidade ao paciente, essa orientação ainda requer estudos adicionais para que se comprove a sua eficácia.

Até o presente momento, o principal fator de risco identificado para a ocorrência de TEV em pacientes com intervenções abaixo do nível do joelho é o histórico de trombose prévia, ${ }^{9-11,24}$ embora seja considerado um fator de risco menos valorizado pelo cirurgião durante a avaliação de risco do paciente ortopédico. ${ }^{17,25} \mathrm{~A}$ identificação deste fator de risco é considerada a única indicação formal para a prescrição de tromboprofilaxia medicamentosa nessa população de pacientes. ${ }^{4}$

Outros fatores considerados de alto risco para a ocorrência de TEV nas cirurgias do tornozelo e do pé são obesidade (índice de massa corporal [IMC] acima de $30 \mathrm{~kg} / \mathrm{m}^{2}$ ), $9,10,18,26$ idade acima dos 50 anos, ${ }^{9-11,17,26}$ e uso de compostos estrogênicos na mulher. $\mathrm{O}$ uso de contraceptivos orais e a terapia de reposição hormonal são fatores de risco clássicos para TEV na população geral, estando presentes em grande parte das pacientes submetidas a cirurgias ou imobilizações no tornozelo e no pé que desenvolvem TEV. ${ }^{9,10,17,19}$ Além disso, fatores de risco genéticos, como a presença do fator $\mathrm{V}$ de Leiden, tipo sanguíneo que não seja 0 , ou níveis elevados de fator VIII devem ser também considerados na avaliação do risco de TEV em cirurgias de pé e de tornozelo, uma vez que eles são fatores de risco conhecidos para cirurgias ortopédicas de outros segmentos. ${ }^{21,27,28}$

Uma questão interessante das doenças do tornozelo e do pé é a influência do local da cirurgia na ocorrência de TEV. Pacientes submetidos a tratamento cirúrgico para lesões do tendão de Aquiles parecem ser aqueles sob maior risco, com a incidência de TEV variando entre 1,36\% e 7,00\%. ${ }^{9,11,18,19} \mathrm{~A}$ incidência de TEV parece também ser aumentada nas afecções do tornozelo $(1,0 \%),{ }^{26}$ retropé $(0,14 \% \text { a } 1,1 \%)^{18,29}$ e antepé $(0,03 \%$ a $4,00 \%){ }^{14,26,30}$

\section{Ferramentas de avaliação de risco}

Um grande desafio para o ortopedista que trata pacientes com lesões no tornozelo e no pé é definir quais pacientes tem maior risco de desenvolver TEV, e, consequentemente, a indicação para tromboprofilaxia medicamentosa. A baixa incidência de TEV nas cirurgias de tornozelo e de pé e a diversidade de fatores de risco e comorbidades relacionadas ao TEV tornam esta tarefa difícil, uma vez que, mesmo raras, as complicações decorrentes do TEV (principalmente da TVP-MMII), como o TEP e a SPT, são extremamente danosas para o paciente. ${ }^{10}$

Diversas ferramentas foram desenvolvidas para avaliar o risco individual de o paciente desenvolver TEV. Saragas et al. $^{14}$ analisaram o formulário Thrombosis Risk Factor Assessment, que pontua os fatores de risco e soma-os para quantificar o risco de TEV. Estes autores avaliaram 216 pacientes com lesões cirúrgicas do tornozelo e do pé, de deformidades do retropé, a fraturas e correção de hálux valgo bilateral. Os autores identificaram que a pontuação média do grupo que desenvolveu TEV foi de 7,7 (variação: 4 a 13 pontos), sendo que $90,9 \%$ destes pacientes somavam 5 ou mais pontos. Assim, os autores recomendam que a pontuação de corte para iniciar a tromboprofilaxia medicamentosa seria de 5 pontos para os pacientes submetidos a cirurgia ortopédica do tornozelo e do pé.

Outra ferramenta utilizada na avaliação de risco de TEV é a do National Institute for Health and Care Excellence (NICE), do Reino Unido. ${ }^{31}$ Uma das vantagens desta ferramenta é a sua capacidade de avaliar todos os pacientes internados, e não somente os cirúrgicos. Além disso, a ferramenta analisa três pontos importantes: a mobilidade do paciente, os fatores de risco para TEV, e o risco de sangramento. A utilidade desta ferramenta foi confirmada em estudos prospectivos em pacientes com fratura de tornozelo, lesões dos ossos do pé, e ruptura do tendão de Aquiles, tratados com imobilização gessada ou cirurgicamente. ${ }^{12,32}$ Entretanto, apesar da grande vantagem de se incluir o risco de sangramento na sua análise, o conceito de mobilidade não é bem definido, podendo gerar interpretações errôneas e decisões equivocadas.

O escore de Caprini, desenvolvido para pacientes cirúrgicos em geral, ${ }^{25,33}$ pontua os fatores de risco relacionados ao paciente e ao tipo de procedimento, sendo considerados de alto risco aqueles que têm pontuação igual ou maior a três pontos (- Figura 1). ${ }^{25}$ Apesar de ainda não ter sido validado para cirurgia ortopédica em geral, o escore de Caprini já foi validado para algumas cirurgias ortopédicas específicas, como fraturas do quadril, ${ }^{34}$ artroplastias coxofemorais e do joelho, ${ }^{35}$ e fraturas em geral. ${ }^{36}$ Além disso, foi também validado para outras áreas cirúrgicas, como cirurgia plástica, ${ }^{37,38}$ otorrinolaringologia, ${ }^{39}$ e ginecologia oncológica. ${ }^{40}$

Assim, o escore de Caprini, por já ter sido validado em diversas áreas cirúrgicas, parece ser o mais confiável e abrangente para uso nas cirurgias ortopédicas em geral. No entanto, o conceito de avaliação de risco trombótico nas cirurgias ortopédicas em geral, e não somente nas cirurgias do quadril e do joelho, carece de difusão na prática clínica. Isso proporcionará melhor predição do risco de TEV e a elaboração de protocolos de tromboprofilaxia mais adequados aos diferentes grupos de maior risco trombótico. 


\begin{tabular}{|c|c|}
\hline \multicolumn{2}{|c|}{ Acrescente 1 ponto para cada situaçăo que se aplique } \\
\hline Idade $41-60$ anos & Infarto agudo do miocárdio \\
\hline Edema MMII & Insuficência cardiaca congestiva ( $<1$ mès) \\
\hline Veias varicosas visivels & Cirurgia maior realizada no úttimo mes \\
\hline Cirurgia menor planejada & História de doença inflamatória intestinal \\
\hline Sepse (<1 mês) & Funçāo pulmonar anormal (DPOC) \\
\hline Sobrepeso ou obesidade (IMC > 25) & Doença pulmonar grave, como pneumonia \\
\hline \multicolumn{2}{|c|}{$\begin{array}{l}\text { Acamado ou com restrição mobilidade, incluindo ortese imobilizador de Ml por menos } \\
\text { de } 72 \mathrm{~h}\end{array}$} \\
\hline \multicolumn{2}{|c|}{ Uso de pílula anticoncepcional ou terapia de reposiçāo hormonal } \\
\hline \multicolumn{2}{|l|}{ Gravidez ou parto no último mes } \\
\hline \multicolumn{2}{|c|}{$\begin{array}{l}\text { História de bebe natimorto, aborto espontaneo recorrente (mais de } 3 \text { ), bebe } \\
\text { prematuro com toxemia ou bebe com restriçăo de crescimento }\end{array}$} \\
\hline \multicolumn{2}{|l|}{ Outros fatores de risco ( 1 ponto cada) $\cdots$} \\
\hline \multicolumn{2}{|c|}{ Acrescente 5 pontos para cada situaçăo que se aplique } \\
\hline \multicolumn{2}{|l|}{$\operatorname{AVC}(<1$ mess) } \\
\hline \multicolumn{2}{|l|}{ Politraumatismo (<1 mês) } \\
\hline \multicolumn{2}{|l|}{ Artroplastia de membros inferiores } \\
\hline \multicolumn{2}{|c|}{ Fratura de quadril, femur e pema ( $<1$ més) } \\
\hline TRM (paralisia) ( $<1$ més) & \\
\hline
\end{tabular}

\begin{tabular}{|l|l|}
\hline \multicolumn{2}{|c|}{ Acrescente 2 pontos para cada situaçăo que se aplique } \\
\hline Idade 61-74 anos & \\
\hline Cancer atual ou no passado & \\
\hline Cirurgia maior planejada estimada em mais de 45 minutos (incluindo laparoscopia) & \\
\hline Bota gessada ou outro dispositivo năo removivel (<1 mês) & \\
\hline Cateter venoso central & \\
\hline Artroscopia & \\
\hline Acamado ou com restriçāo mobilidade por mais de 72h & \\
\hline \multicolumn{1}{|c|}{ Acrescente 3 pontos para cada situaçăio que se aplique } \\
\hline Idade 75 anos ou mais & \\
\hline Trombose previa (TVP ou embolia pulmonar) & \\
\hline História familiar de trombose & \\
\hline Fator V de Leiden positivo & \\
\hline Protrombina positivo & \\
\hline Anticoagulante lúpico positivo & \\
\hline Trombocitopenia induzida por heparina & \\
\hline Homocisteina sérica elevada & \\
\hline Anticorpos anticardiolipina elevados & \\
\hline Outra trombofilia adquirida ou congenita & \\
\hline
\end{tabular}

Fig. 1 Escore de Caprini. Abreviações: AVC, acidente vascular cerebral; DPOC, doença pulmonar obstrutiva crônica; IMC, índice de massa corporal; MMII, membros inferiores; h, horas; TEV, tromboembolismo venoso; TRM, trauma raquimedular; TVP, trombose venosa profunda. Fonte: Traduzido e adaptado de Caprini JA. Risk assessment as a guide for the prevention of the many faces of venous thromboembolism. Am J Surg 2010;199 (1 Suppl):S3-10.25

\section{Tipos de tromboprofilaxia}

\section{Mecânica}

O princípio da tromboprofilaxia mecânica se baseia no uso da compressão gradativa da musculatura sobre as veias, potencializando o retorno venoso. ${ }^{16}$ A tromboprofilaxia mecânica nas cirurgias ortopédicas pode ser realizada utilizando dispositivos de compressão pneumática intermitente (DCPI) e meias de compressão elásticas. ${ }^{41}$ Em recente meta-análise ${ }^{42}$ com 16.164 pacientes, o uso de DCPI se mostrou eficaz na redução de TEV em pacientes internados quando comparado ao uso de placebo, sendo o risco relativo (RR) para TVP de 0,43 (IC95\%: 0,36-0,52), e, para TEP, de 0,48 (IC95\%: 0,33-0,69). ${ }^{42}$ Como vantagens, esses dispositivos não têm os efeitos adversos apresentados pelas drogas anticoagulantes, tais como sangramento, e tampouco requerem monitoramento laboratorial. ${ }^{43}$ No entanto, apresentam como importante desvantagem a dificuldade de adesão do paciente no período pós-operatório, uma vez que muitos desses indivíduos submetidos a cirurgias no tornozelo e no pé ficam imobilizados, sendo incapazes de utilizar estes tipos de dispositivos no membro operado. Assim, seu uso fica reservado ao membro contralateral durante o período de internação. Ressalta-se que o método mecânico mais simples de prevenção de TEV é a deambulação precoce. ${ }^{22,43}$

\section{Medicamentosa}

O uso de medicamentos para a profilaxia de TEV (profilaxia medicamentosa) nas cirurgias do tornozelo e do pé é mais difundido do que a profilaxia mecânica. Isso se deve a questões relacionadas à menor disponibilidade do dispositivo de compressão, ao preço das meias, à menor facilidade de uso e a fatores locais, tais como a necessidade de imobi- lização do membro inferior no pós-operatório de cirurgias de tornozelo e de pé.

Entre os medicamentos aprovados para tromboprofilaxia nas cirurgias ortopédicas estão o ácido acetilsalicílico (AAS), as heparinas (não fracionada - HNF, e de baixo peso molecular - HBPM), os anticoagulantes orais de ação direta, e a varfarina. Caso os pacientes estejam utilizando essas medicações antes da cirurgia, é importante observar o período necessário de interrupção previamente à cirurgia para evitar sangramentos (-Tabela 1). Cada um desses medicamentos age em uma determinada etapa da via da coagulação, inibindo diferentes fatores (-Figura 2).

O AAS é um inibidor da ciclooxigenase, que promove a diminuição da síntese de prostaglandinas e tromboxano, inibindo assim a agregação plaquetária. É um medicamento de fácil acesso, barato, e com longa experiência de uso. Apesar da recomendação sobre o uso do AAS como agente único para tromboprofilaxia ser controversa na literatura, estudos recentes ${ }^{44}$ demonstram que nas cirurgias de artroplastia do quadril e do joelho, ele pode ser usado após um breve ciclo de cinco dias de um anticoagulante oral de ação direta (rivaroxabana). ${ }^{45,46}$ Estudos sobre o uso do ácido acetilsalicílico como tromboprofilático em cirurgias do tornozelo e do pé ainda são escassos, conforme observado em recente consenso sobre o assunto. ${ }^{17}$

A HNF e a HBPM têm como alvo a inibição de várias proteases da coagulacão (-Figura 2). A HNF age indiretamente, ligando-se à antitrombina (um anticoagulante natural), potencializando a ação inibitória desta sobre várias proteases da coagulação, incluindo a trombina (fator Ila) e o fator Xa. ${ }^{41}$ As HBPMs se originam da digestão parcial da HNF, resultando em menor ligação a outras proteínas plasmáticas e maior especificidade de ação contra o fator Xa. Ambas as formas são 
Tabela 1 Medicamentos mais utilizados na profilaxia do tromboembolismo venoso nas cirurgias ortopédicas

\begin{tabular}{|c|c|c|c|}
\hline Droga & Posologia & Tempo de interrupção antes da cirurgia & Observação \\
\hline $\begin{array}{l}\text { Ácido } \\
\text { acetilsalicílico }\end{array}$ & 100 mg/dia dose única, via oral & 5 a 10 dias antes da cirurgia & $\begin{array}{l}\text { Não deve ser utilizada como monoterapia. } \\
\text { Usar como terapia estendida após cinco dias } \\
\text { de rivaroxabana em pacientes de baixo risco } \\
\text { trombótico }\end{array}$ \\
\hline $\begin{array}{l}\text { HBPM - } \\
\text { Enoxaparina }\end{array}$ & 40 mg/dia dose única, subcutânea & $\begin{array}{l}12 \text { horas se baixo risco de sangramento; } \\
24 \text { horas se alto risco }\end{array}$ & Iniciar 12 horas após a cirurgia \\
\hline $\begin{array}{l}\text { HBPM - } \\
\text { Dalteparina }\end{array}$ & 5.000 UI/dia dose única, subcutânea & $\begin{array}{l}12 \text { horas se baixo risco de sangramento; } \\
24 \text { horas se alto risco }\end{array}$ & Iniciar 12 horas após a cirurgia \\
\hline $\begin{array}{l}\text { HBPM - } \\
\text { Nadroparina }\end{array}$ & $\begin{array}{l}38 \text { Ul anti-Xa por kilo de peso corporal } \\
(0.2 \text { a } 0.4 \mathrm{~mL}) \text {; aumentar em } 50 \% \text { no quarto } \\
\text { dia do pós-operatório }(0.3 \text { a } 0.6 \mathrm{~mL}) \text {. }\end{array}$ & $\begin{array}{l}12 \text { horas se baixo risco de sangramento; } \\
24 \text { horas se alto risco }\end{array}$ & Iniciar 12 horas após a cirurgia \\
\hline Rivaroxabana & $10 \mathrm{mg} / \mathrm{dia}$, via oral & $\begin{array}{l}24 \text { horas se baixo risco de sangramento; } \\
48 \text { horas se risco moderado ou alto, ou } \\
\text { paciente idoso }\end{array}$ & Iniciar $>6$ horas após a cirurgia \\
\hline Dabigatrana & $220 \mathrm{mg} / \mathrm{dia}$, via oral & $\begin{array}{l}48 \text { a } 72 \text { horas se função renal normal; } \\
\text { mínimo de } 5 \text { dias para cirurgias ortopéd- } \\
\text { icas maiores }\end{array}$ & $\begin{array}{l}\text { Iniciar com } 110 \mathrm{mg} \text { administrados } 1-4 \text { horas } \\
\text { após a cirurgia, e a seguir com } 220 \mathrm{mg} \text { uma } \\
\text { vez ao dia }\end{array}$ \\
\hline Apixabana & $2,5 \mathrm{mg}$ duas vezes ao dia, via oral & $\begin{array}{l}24 \text { horas se baixo risco para sangramento; } \\
48 \text { horas se risco moderado ou alto }\end{array}$ & Iniciar $\geq 12$ horas após a cirurgia \\
\hline Varfarina & Na maioria dos casos, 5-10 mg/dia, via oral & 3 a 5 dias & $\begin{array}{l}\text { Após o terceiro dia, ajustar conforme RNI com } \\
\text { alvo terapêutico de } 1,5 \text { a } 2,5 \text {. Iniciar } 12 \text { a } 24 \\
\text { horas após a cirurgia. Sugere-se usar um } \\
\text { nomograma de ajuste de dose de varfarina } \\
\text { validado }\end{array}$ \\
\hline
\end{tabular}

Abreviações: mg, miligramas; HBPM, heparina de baixo peso molecular; RNI, razão normalizada internacional.

eficazes, seguras e administradas por via subcutânea. Entretanto, as HBPMs têm maior praticidade de uso (dose única diária), menor risco de plaquetopenia induzida por heparina, e menor indução de osteopenia. ${ }^{47}$ Assim, a HBPM é o anticoagulante mais recomendada para a profilaxia de TEV nas cirurgias do tornozelo e do pé, inclusive pelas diretrizes mais recentes, ${ }^{9,17}$ sendo, até o momento, a droga de escolha para pacientes sob risco de TEV, tanto cirúrgicos quanto não cirúrgicos, desde que estejam imobilizados. ${ }^{17}$

Os anticoagulantes orais de ação direta (AOADs) são uma nova classe de anticoagulantes de ação específica, de uso oral, direcionados contra o fator Xa (apixabana, rivaroxabana, edoxabana, betrixabana) ou o fator Ila (dabigatrana). Têm como principal vantagem a falta de necessidade do monitoramento periódico por meio de testes laboratoriais e de administração parenteral/subcutânea. Além disso, são drogas mais seguras em comparação com a varfarina quanto à ocorrência de sangramento grave, embora o sangramento gastrointestinal seja mais comum com o seu uso.

A rivaroxabana, apixabana e dabigatrana foram aprovadas para profilaxia de TEV nas artroplastias do joelho e do quadril. ${ }^{48,49}$ Entretanto, nenhuma delas foi autorizada para uso em fraturas do fêmur proximal e do quadril. ${ }^{4,7,50}$ Apesar da aprovação de uso dos AOADs nas grandes cirurgias ortopédicas, até o presente momento existem dados referentes apenas à rivaroxabana na profilaxia de TEV em cirurgias abaixo do nível do joelho. Em um estudo randomizado com 3.604 pacientes em um grupo utilizando $40 \mathrm{mg}$ de enoxaparina subcutânea e outro utilizando $10 \mathrm{mg}$ de rivaroxabana por via oral, ambas sendo administradas uma vez ao dia, a rivaroxabana se mostrou mais eficaz na prevenção de TEV em comparação com a enoxaparina (RR: 0,25; IC95\%: $0,09-0,75)$, sem aumento do risco de sangramento. ${ }^{51}$
A varfarina é um antagonista da vitamina $\mathrm{K}$ que inibe a síntese de fatores de coagulação dependentes de cálcio - II, VII, IX e X -, bem como das proteínas C, S e Z. ${ }^{41}$ A dose recomendada é variável, sendo na maioria dos casos entre $5 \mathrm{mg}$ e $10 \mathrm{mg}$ diariamente no período pós-operatório. ${ }^{41}$ Apesar de também ser uma droga barata e de grande disponibilidade, somente deve ser usada como agente tromboprofilático nas cirurgias ortopédicas caso não exista acesso às heparinas ou aos AOADs. A varfarina requer um monitoramento contínuo do tempo da protrombina e de seu derivado, a relação normatizada internacional (RNI), que deve ter como alvo um valor entre dois e três para ser considerada uma anticoagulação adequada para a prevenção do TEV. Sua meia-vida média é de 2,5 dias, o que faz com que deva ser suspensa pelo menos 3 dias antes de uma intervenção cirúrgica. ${ }^{52}$

\section{Tempo de uso da tromboprofilaxia}

A tromboprofilaxia medicamentosa deve ser iniciada no período pós-operatório, 12 horas após a cirurgia se o paciente não apresentar sangramento ativo ou alto risco de sangramento. ${ }^{53}$

O tempo de manutenção da tromboprofilaxia medicamentosa em determinadas cirurgias ortopédicas é bem definido. Esse é o caso das grandes cirurgias ortopédicas (artroplastias de quadril e joelho), cuja anticoagulação no período pós-operatório deve ser de no mínimo 10 a 14 dias, podendo atingir até 35 dias. ${ }^{4} \mathrm{~A}$ anticoagulação poderá ser suspensa até que o paciente esteja deambulando, desde que tenha sido administrada por no mínimo entre 10 e 14 dias. No entanto, a simples replicação desses prazos nas cirurgias do tornozelo e do pé não é adequada. 


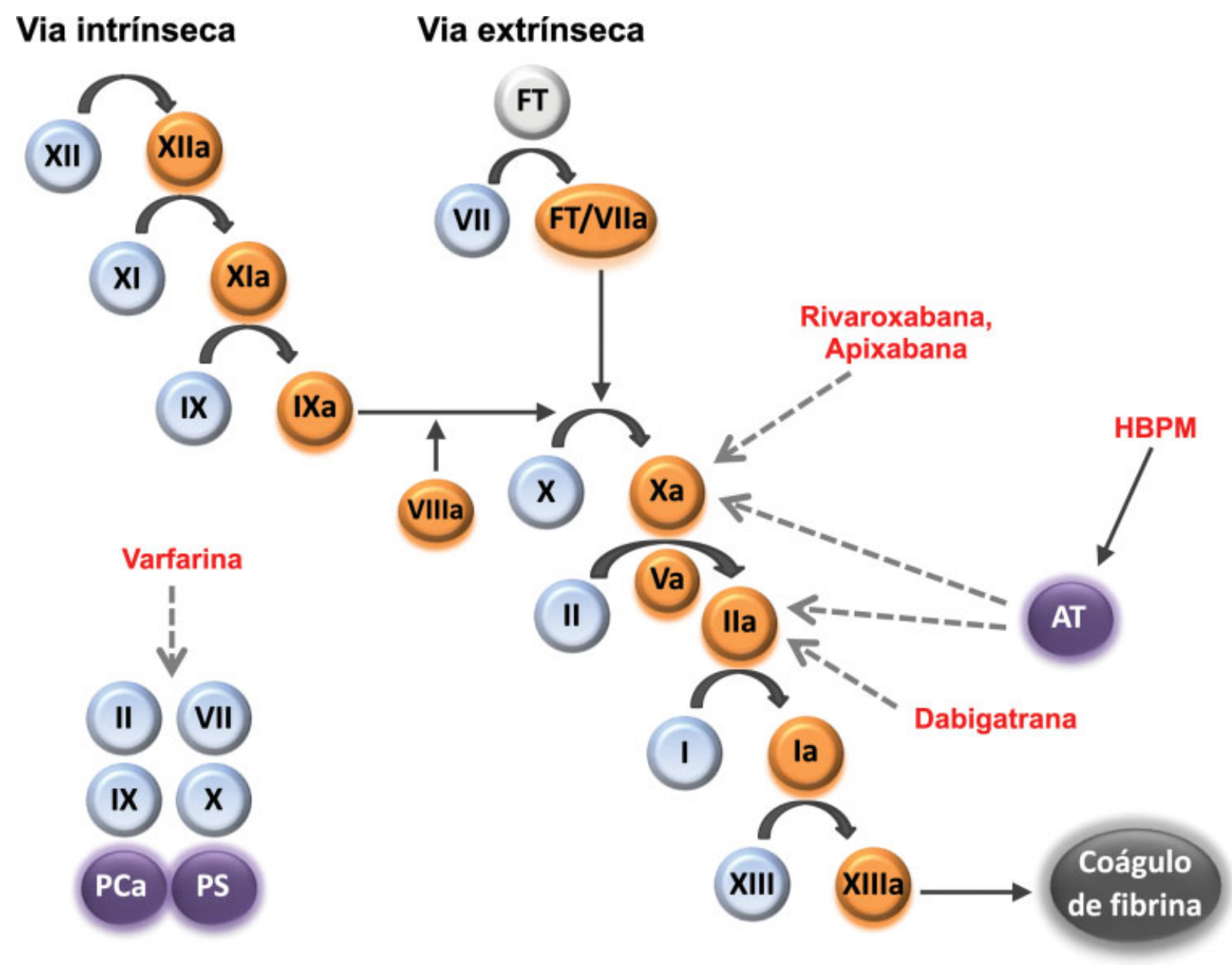

Fig. 2 Representação esquemática do controle da coagulação e indicação dos sítios de atuação dos principais anticoagulantes. Abreviações: a, ativado; AT, antitrombina; FT, fator tecidual; HBPM: heparina de baixo peso molecular.

O uso de algum tipo de imobilização no tratamento das doenças do tornozelo e do pé está associado à ocorrência de TEV em $4,3 \%$ a $40,0 \%$ dos pacientes, sejam eles tratados cirurgicamente ou não. ${ }^{54} \mathrm{O}$ fato de o paciente permanecer sem apoiar o membro inferior no solo ou mesmo com apoio parcial também atuam como fatores de risco para a ocorrência de TVP. ${ }^{23,55} \mathrm{Com}$ isso, imobilização e o fato de estar sem apoiar o pé no chão são fatores que devem ser considerados no momento de se definir o tempo de profilaxia.

De forma geral, é consenso entre especialistas que a profilaxia deva ser mantida durante todo o período em que o paciente estiver imobilizado, ${ }^{17}$ ou pelo menos enquanto estiver sem apoio e imobilizado. A suspensão da profilaxia poderá ocorrer assim que o apoio do membro inferior tratado ocorrer ou após a retirada da imobilização mesmo que sem apoio, o que permitirá a contração ativa da musculatura da panturrilha, diminuindo o risco de TEV. ${ }^{14}$

\section{Complicações}

O grande debate sobre a profilaxia reside no fato da potencial ocorrência de complicações decorrentes da administração de agentes anticoagulantes, principalmente em grandes cirurgias. Entretanto, informações sobre a ocorrência de compli- cações relacionadas ao uso de profilaxia medicamentosa nas cirurgias do tornozelo e do pé são escassas.

Entre as complicações descritas nas cirurgias dos membros inferiores após o uso de anticoagulantes estão os sangramentos, que ocorrem de $0,3 \%$ a $1 \%$ dos $\operatorname{casos}^{49,56}$ Os fatores associados ao aumento do sangramento pós-operatório neste grupo de pacientes são as cirurgias de revisão, sangramento perioperatório superior ao esperado, dissecção agressiva de partes moles, sangramento gastrointestinal ou geniturinário recentes, terapia antiagregante concomitante, e doença renal avançada. ${ }^{57}$

Outras complicações locais possíveis são hematomas, ${ }^{49}$ que podem levar a deiscência e infecção na ferida operatória, que muitas vezes são dramáticas nas cirurgias do tornozelo e do pé. Além disso, a trombocitopenia induzida por heparina, complicação considerada grave por aumentar os riscos de eventos trombóticos, é mais comum em pacientes cirúrgicos, com uma taxa de incidência de $0,20 \%$ a $0,36 \%$ nos pacientes tratados com HBPM. ${ }^{58,59}$

\section{Considerações Finais}

O TEV é uma das complicações do tratamento das condições do tornozelo e do pé. Embora sua incidência seja menor do que nas cirurgias de quadril e de joelho, ainda são necessários 
estudos robustos que avaliem fatores de risco concomitantes que atuem de forma interativa e aumentem o risco de TEV. A avaliação dos fatores de risco juntamente com o tipo de cirurgia ortopédica deve ser individualizada para embasar e focar a tromboprofilaxia em grupos de maior risco. Os estudos atualmente existentes carecem de evidências para a recomendação universal de tromboprofilaxia nas cirurgias de tornozelo e de pé.

Uma vez indicada tromboprofilaxia medicamentosa, a HBPM (mais comumente, a enoxaparina) é o anticoagulante de eleição na profilaxia de TEV nos pacientes com intervenções no tornozelo eno pé. O uso dos AOADs como agentes tromboprofiláticos nas cirurgias ortopédicas de lesões abaixo do nível do joelho ainda carece de mais estudos. No presente, apenas a rivaroxabana mostrou-se eficaz e segura, e obteve aprovação para o uso. Os demais AOADs, conforme a orientação das diretrizes até o momento, devem ser utilizados somente se houver recusa do paciente para o uso de heparina, e se houver indisponibilidade da rivaroxabana. A tromboprofilaxia deve ser mantida durante todo o período em que o paciente estiver sem apoiar o membro ou estiver imobilizado, uma vez que estes são fatores reconhecidamente de risco para a ocorrência de TEV.

Estudos futuros deverão focar no efeito de interação de outros fatores de risco concomitantes aos procedimentos ortopédicos de tornozelo e de pé no desenvolvimento de TEV, bem como na validação e no aprimoramento das ferramentas de avaliação de risco e sua automatização. Isso permitirá identificar o paciente com maior risco, e propiciará a adequada indicação de profilaxia, com redução de custos e complicações.

\section{Suporte Financeiro}

Não houve suporte financeiro de fontes públicas, comerciais, ou sem fins lucrativos.

\section{Conflito de Interesses}

Os autores declaram não haver conflito de interesses.

\section{Referências}

1 Geerts WH, Bergqvist D, Pineo GF, et al. Prevention of venous thromboembolism: American College of Chest Physicians Evidence-Based Clinical Practice Guidelines (8th Edition). Chest 2008;133(6 Suppl):381S-453S

2 Naess IA, Christiansen SC, Romundstad P, Cannegieter SC, Rosendaal FR, Hammerstrøm J. Incidence and mortality of venous thrombosis: a population-based study. J Thromb Haemost 2007;5(04):692-699

3 Anderson FA Jr, Spencer FA. Risk factors for venous thromboembolism. Circulation 2003;107(23, Suppl 1):I9-I16

4 Falck-Ytter Y, Francis CW, Johanson NA, et al. Prevention of VTE in orthopedic surgery patients: Antithrombotic Therapy and Prevention of Thrombosis, 9th ed: American College of Chest Physicians Evidence-Based Clinical Practice Guidelines. Chest 2012; 141(2 Suppl):e278S-e325S

5 Dai WL, Lin ZM, Shi ZJ, Wang J. Venous Thromboembolic Events after Total Knee Arthroplasty: Which Patients Are at a High Risk? J Knee Surg 2020;33(10):947-957

6 Senay A, Trottier M, Delisle J, et al. Incidence of symptomatic venous thromboembolism in 2372 knee and hip replacement patients after discharge: data from a thromboprophylaxis registry in Montreal, Canada. Vasc Health Risk Manag 2018;14:81-89

7 Anderson DR, Morgano GP, Bennett C, et al. American Society of Hematology 2019 guidelines for management of venous thromboembolism: prevention of venous thromboembolism in surgical hospitalized patients. Blood Adv 2019;3(23):3898-3944

8 Jacobs JJ, Mont MA, Bozic KJ, et al. American Academy of Orthopaedic Surgeons clinical practice guideline on: preventing venous thromboembolic disease in patients undergoing elective hip and knee arthroplasty. J Bone Joint Surg Am 2012;94(08): 746-747

9 Calder JD, Freeman R, Domeij-Arverud E, van Dijk CN, Ackermann PW. Meta-analysis and suggested guidelines for prevention of venous thromboembolism (VTE) in foot and ankle surgery. Knee Surg Sports Traumatol Arthrosc 2016;24(04):1409-1420

10 Richey JM, Ritterman Weintraub ML, Schuberth JM. Incidence and Risk Factors of Symptomatic Venous Thromboembolism Following Foot and Ankle Surgery. Foot Ankle Int 2019;40(01):98-104

11 Pedersen MH, Wahlsten LR, Grønborg H, Gislason GH, Petersen MM, Bonde AN. Symptomatic Venous Thromboembolism After Achilles Tendon Rupture: A Nationwide Danish Cohort Study of 28,546 Patients With Achilles Tendon Rupture. Am J Sports Med 2019;47(13):3229-3237

12 Blanco JA, Slater G, Mangwani J. A Prospective Cohort Study of Symptomatic Venous Thromboembolic Events in Foot and Ankle Trauma: The Need for Stratification in Thromboprophylaxis? J Foot Ankle Surg 2018;57(03):484-488

13 Sullivan M, Eusebio ID, Haigh K, Panti JP, Omari A, Hang JR. Prevalence of Deep Vein Thrombosis in Low-Risk Patients After Elective Foot and Ankle Surgery. Foot Ankle Int 2019;40(03):330-335

14 Saragas NP, Ferrao PNF, Saragas E, Jacobson BF. The impact of risk assessment on the implementation of venous thromboembolism prophylaxis in foot and ankle surgery. Foot Ankle Surg 2014;20 (02):85-89

15 Parisi R, Visonà A, Camporese G, et al. Isolated distal deep vein thrombosis: efficacy and safety of a protocol of treatment. Treatment of Isolated Calf Thrombosis (TICT) Study. Int Angiol 2009;28(01):68-72

16 Chien BY, Dixon T, Guss D, DiGiovanni C. Venous Thromboembolism Disease Prophylaxis in Foot and Ankle Surgery. Orthop Clin North Am 2018;49(02):265-276

17 Fleischer AE, Abicht BP, Baker JR, Boffeli TJ, Jupiter DC, Schade VL. American College of Foot and Ankle Surgeons' clinical consensus statement: risk, prevention, and diagnosis of venous thromboembolism disease in foot and ankle surgery and injuries requiring immobilization. J Foot Ankle Surg 2015;54(03):497-507

18 Ahmad J, Lynch MK, Maltenfort M. Incidence and Risk Factors of Venous Thromboembolism After Orthopaedic Foot and Ankle Surgery. Foot Ankle Spec 2017;10(05):449-454

19 Mangwani J, Sheikh N, Cichero M, Williamson D. What is the evidence for chemical thromboprophylaxis in foot and ankle surgery? Systematic review of the English literature. Foot 2015; 25(03):173-178

20 Rosendaal FR. Venous thrombosis: a multicausal disease. Lancet 1999;353(9159):1167-1173

21 van Adrichem RA, Debeij J, Nelissen RG, Schipper IB, Rosendaal FR, Cannegieter SC. Below-knee cast immobilization and the risk of venous thrombosis: results from a large population-based casecontrol study. J Thromb Haemost 2014;12(09):1461-1469

22 Healy B, Beasley R, Weatherall M. Venous thromboembolism following prolonged cast immobilisation for injury to the tendo Achillis. J Bone Joint Surg Br 2010;92(05):646-650

23 Riou B, Rothmann C, Lecoules N, et al. Incidence and risk factors for venous thromboembolism in patients with nonsurgical isolated lower limb injuries. Am J Emerg Med 2007;25(05):502-508

24 Nemeth B, Timp JF, van Hylckama Vlieg A, Rosendaal FR, Cannegieter SC. High risk of recurrent venous thrombosis in patients with lowerleg cast immobilization.J Thromb Haemost 2018;16(11):2218-2222 
25 Caprini JA. Risk assessment as a guide for the prevention of the many faces of venous thromboembolism. Am J Surg 2010;199(1, Suppl)S3-S10

26 Huntley SR, Abyar E, Lehtonen EJ, Patel HA, Naranje S, Shah A. Incidence of and Risk Factors for Venous Thromboembolism After Foot and Ankle Surgery. Foot Ankle Spec 2019;12(03):218-227

27 van Adrichem RA, Nelissen RG, Schipper IB, Rosendaal FR, Cannegieter SC. Risk of venous thrombosis after arthroscopy of the knee: results from a large population-based case-control study. J Thromb Haemost 2015;13(08):1441-1448

28 Zhou X, Qian W, Li J, et al. Who are at risk for thromboembolism after arthroplasty? A systematic review and meta-analysis. Thromb Res 2013;132(05):531-536

29 Jameson SS, Augustine A, James P, et al. Venous thromboembolic events following foot and ankle surgery in the English National Health Service. J Bone Joint Surg Br 2011;93(04):490-497

30 Radl R, Kastner N, Aigner C, Portugaller H, Schreyer H, Windhager R. Venous thrombosis after hallux valgus surgery. J Bone Joint Surg Am 2003;85(07):1204-1208

31 Department of Health VTE risk assessment tool. National Institute of Health and Care Excellence (NICE), published date: 21 March 2018. Available from: https://www.nice.org.uk/guidance/ ng89/chapter/Recommendations\#risk-assessment

32 Haque S, Bishnoi A, Khairandish H, Menon D. Thromboprophylaxis in Ambulatory Trauma Patients With Foot and Ankle Fractures: Prospective Study Using a Risk Scoring System. Foot Ankle Spec 2016;9(05):388-393

33 Caprini JA. Thrombosis risk assessment as a guide to quality patient care. Dis Mon 2005;51(2-3):70-78

34 Luksameearunothai K, Sa-Ngasoongsong P, Kulachote N, et al. Usefulness of clinical predictors for preoperative screening of deep vein thrombosis in hip fractures. BMC Musculoskelet Disord 2017;18(01):208

35 Krauss ES, Segal A, Cronin M, et al. Implementation and Validation of the 2013 Caprini Score for Risk Stratification of Arthroplasty Patients in the Prevention of Venous Thrombosis. Clin Appl Thromb Hemost 2019;25:1076029619838066

36 Dashe J, Parisien RL, Pina M, De Giacomo AF, Tornetta P 3rd. Is the Caprini Score Predictive of Venothromboembolism Events in Orthopaedic Fracture Patients? J Orthop Trauma 2019;33(06): 269-275

37 Pannucci CJ, Bailey SH, Dreszer G, et al. Validation of the Caprini risk assessment model in plastic and reconstructive surgery patients. J Am Coll Surg 2011;212(01):105-112

38 Swanson E. Caprini Scores, Risk Stratification, and Rivaroxaban in Plastic Surgery: Time to Reconsider Our Strategy. Plast Reconstr Surg Glob Open 2016;4(06):e733

39 Shuman AG, Hu HM, Pannucci CJ, Jackson CR, Bradford CR, Bahl V. Stratifying the risk of venous thromboembolism in otolaryngology. Otolaryngol Head Neck Surg 2012;146(05):719-724

40 Stroud W, Whitworth JM, Miklic M, et al. Validation of a venous thromboembolism risk assessment model in gynecologic oncology. Gynecol Oncol 2014;134(01):160-163

41 Carr P, Ehredt DJ Jr, Dawoodian A. Prevention of Deep Venous Thromboembolism in Foot and Ankle Surgery. Clin Podiatr Med Surg 2019;36(01):21-35

42 Ho KM, Tan JA. Stratified meta-analysis of intermittent pneumatic compression of the lower limbs to prevent venous throm- boembolism in hospitalized patients. Circulation 2013;128(09): 1003-1020

43 Leme LE, Sguizzatto GT. Prophylaxis of venous thromboembolism in orthopaedic surgery. Rev Bras Ortop 2015;47(06):685-693

44 Anderson DR, Dunbar M, Murnaghan J, et al. Aspirin or Rivaroxaban for VTE Prophylaxis after Hip or Knee Arthroplasty. N Engl J Med 2018;378(08):699-707

45 Chu JN, Maselli J, Auerbach AD, Fang MC. The risk of venous thromboembolism with aspirin compared to anticoagulants after hip and knee arthroplasty. Thromb Res 2017;155:65-71

46 Deirmengian GK, Heller S, Smith EB, Maltenfort M, Chen AF, Parvizi J. Aspirin Can Be Used as Prophylaxis for Prevention of Venous Thromboembolism After Revision Hip and Knee Arthroplasty. J Arthroplasty 2016;31(10):2237-2240

47 Flevas DA, Megaloikonomos PD, Dimopoulos L, Mitsiokapa E, Koulouvaris P, Mavrogenis AF. Thromboembolism prophylaxis in orthopaedics: an update. EFORT Open Rev 2018;3(04):136-148

48 Eriksson BI, Borris LC, Friedman RJ, et al. RECORD1 Study Group. Rivaroxaban versus enoxaparin for thromboprophylaxis after hip arthroplasty. N Engl J Med 2008;358(26):2765-2775

49 Lassen MR, Ageno W, Borris LC, et al. RECORD3 Investigators. Rivaroxaban versus enoxaparin for thromboprophylaxis after total knee arthroplasty. N Engl J Med 2008;358(26):2776-2786

50 Raskob GE, Gallus AS, Pineo GF, et al. Apixaban versus enoxaparin for thromboprophylaxis after hip or knee replacement: pooled analysis of major venous thromboembolism and bleeding in 8464 patients from the ADVANCE- 2 and ADVANCE- 3 trials. J Bone Joint Surg Br 2012;94(02):257-264

51 Samama CM, Laporte S, Rosencher N, et al. PRONOMOS Investigators. Rivaroxaban or Enoxaparin in Nonmajor Orthopedic Surgery. N Engl J Med 2020;382(20):1916-1925

52 Ageno W, Gallus AS, Wittkowsky A, Crowther M, Hylek EM, Palareti G. Oral anticoagulant therapy: Antithrombotic Therapy and Prevention of Thrombosis, 9th ed: American College of Chest Physicians Evidence-Based Clinical Practice Guidelines. Chest 2012;141(2 Suppl):e44S-e88S

53 Strebel N, Prins M, Agnelli G, Büller HR. Preoperative or postoperative start of prophylaxis for venous thromboembolism with low-molecular-weight heparin in elective hip surgery? Arch Intern Med 2002;162(13):1451-1456

54 Testroote M, Stigter W, de Visser DC, Janzing H. Low molecular weight heparin for prevention of venous thromboembolism in patients with lower-leg immobilization. Cochrane Database Syst Rev 2008;(04):CD006681

55 Hanslow SS, Grujic L, Slater HK, Chen D. Thromboembolic disease after foot and ankle surgery. Foot Ankle Int 2006;27(09):693-695

56 Mayle RE Jr, DiGiovanni CW, Lin SS, Tabrizi P, Chou LB. Current concepts review: venous thromboembolic disease in foot and ankle surgery. Foot Ankle Int 2007;28(11):1207-1216

57 Francis CW. Prevention of VTE in patients having major orthopedic surgery. J Thromb Thrombolysis 2013;35(03):359-367

58 Bloemen A, Testroote MJ, Janssen-Heijnen ML, Janzing HM. Incidence and diagnosis of heparin-induced thrombocytopenia (HIT) in patients with traumatic injuries treated with unfractioned or low-molecularweight heparin: a literature review. Injury 2012;43(05):548-552

59 Martel N, Lee J, Wells PS. Risk for heparin-induced thrombocytopenia with unfractionated and low-molecular-weight heparin thromboprophylaxis: a meta-analysis. Blood 2005;106(08):2710-2715 